\title{
UIUC Collector Erosion and Optical Lifetime Project Results: Time Dependent Exposures
}

\author{
Darren A. Alman ${ }^{\mathrm{a}}$, Huatan Qiu ${ }^{\mathrm{a}}$, Keith C. Thompson ${ }^{\mathrm{a}}$, Erik L. Antonsen ${ }^{\mathrm{a}}$, Joshua B. Spencer ${ }^{\mathrm{a}}$, \\ Matthew R. Hendricks ${ }^{\mathrm{a}}$, Brian E. Jurczyk ${ }^{\mathrm{a}}$, David N. Ruzic ${ }^{\mathrm{a}}$, Timothy Spila ${ }^{\mathrm{b}}$, Ginger Edwards ${ }^{\mathrm{c}}$, \\ Stefan Wurm ${ }^{\mathrm{c}}$, Obert Wood ${ }^{\mathrm{c}}$, Robert Bristol ${ }^{\mathrm{d}}$ \\ ${ }^{a}$ University of Illinois at Urbana-Champaign, Urbana, IL 61801 USA \\ ${ }^{\mathrm{b}}$ Center for Microanalysis of Materials, University of Illinois at Urbana-Champaign, IL 61801 USA \\ ${ }^{\mathrm{c}}$ SEMATECH, Austin, TX 78741 USA \\ ${ }^{\mathrm{d} I n t e l}$ Corporation, Portland, OR 97123 USA
}

\begin{abstract}
Extreme ultraviolet (EUV) light sources are needed for next-generation lithography. A critical consideration in the development of such a source is the lifetime of collector optics. Frequent replacement of the mirror system will detract from the economic feasibility of EUV lithography. The Xtreme Commercial EUV Exposure Device (XCEED) at the University of Illinois has been designed to test the performance of various EUV mirror materials during operation of a commercial EUV source, and to investigate the mechanisms behind observed losses in reflectivity over a varying number of shots. Recently, four Mo/Si multilayer mirror samples were exposed in XCEED for variable numbers of shots, up to 40 million. The samples were analyzed to determine how the surface roughness was effected and how much material was eroded vs. time. XCEED also includes photodiodes to measure light output and light reflected from mirror samples, as well as a time-of-flight energy sector analyzer (TOF-ESA) for debris characterization. The results of these timedependent exposures are presented in this work.
\end{abstract}

Keywords: Condenser Optics Lifetime, EUV Time Exposure, Damage Characterization

\section{INTRODUCTION}

Current lithography using $193 \mathrm{~nm}$ light can be extended down to the 65 and even $45 \mathrm{~nm}$ nodes using various resolution enhancement techniques. Beyond that however, next generation lithography methods, e.g. extreme ultraviolet lithography (EUVL), will be needed to continue producing smaller and faster chips around the end of the decade. EUV light output from a pulsed plasma source will need to be collected and focused on the mask using a series of mirrors. For EUV to be economically feasible, these mirror components must have a reasonable lifetime before they need to be replaced. Frequent replacement would lead to an unacceptable amount of downtime of the lithography tool.

Both DPP and LPP sources produce strong electric fields during plasma expansion that can accelerate the fuel ions to extremely high energies, on the order of tens of $\mathrm{keV}$ for xenon and perhaps $1 \mathrm{keV}$ for lighter ions like lithium. The implication of this is that the mirror surfaces will be bombarded by a potentially large flux of energetic ions, eventually destroying the reflective properties of the mirror. The mechanisms for mirror degradation could be surface erosion, roughening, deposition or implantation of impurities, and layer mixing. The Xtreme Commercial EUV Exposure Device (XCEED) at the University of Illinois has been developed to test the performance of various EUV mirror materials during operation of a commercial EUV source, and to investigate the mechanisms behind observed losses in reflectivity over a varying number of shots.

The XCEED experiment consists of an XTS 13-35 commercial EUV source mated to a custom designed test chamber. The source, manufactured by Xtreme Technologies $\mathrm{GmbH}$, is a z-pinch device using xenon gas. A debris mitigation system, manufactured by Xtreme Technologies, is located between the pinch location and the location of the samples and diagnostics. The samples are located 22 inches $(\sim 56 \mathrm{~cm})$ from the pinch, on sample holders that can hold up to sixteen $1 / 2$ " $x 1 / 2$ " square mirror samples each in either a normal incidence or grazing incidence configuration. The four sample 
holders can be individually removed from the test chamber without breaking vacuum through a load lock. The main diagnostics are photodiodes and debris detectors.

Two IRD Ti/Zr/Si 6/480/50 $\mathrm{nm}$ photodiodes are used in combination to measure changes in reflectivity of mirror samples. One is a reference photodiode located at $35^{\circ}$ and far from the pinch location to minimize debris, but still with a direct line of sight to the pinch. To measure only in-band EUV output from the source, this photodiode setup uses a one bounce configuration off of a 40 bilayer $\mathrm{Mo} / \mathrm{Si}$ mirror with a $4 \mathrm{~nm} \mathrm{Si} \mathrm{capping} \mathrm{layer} \mathrm{mirror} \mathrm{(70 \%} \mathrm{reflectivity).} \mathrm{To} \mathrm{further}$ limit damage to this mirror, the photodiode is housed behind a $1 \mathrm{~mm}$ orifice and backfilled with He gas. The EUV reflected from the samples is measured with a second photodiode that is on an adjustable mount so that it can rotate $360^{\circ}$ inside the chamber to look at the four different sample mounts. It was designed to be looking away from the pinch, and shielded from both direct light from the pinch and debris. The only signal should come from reflection of the sample that the photodiode is aimed at. After the results of the first exposures with this setup, however, this design will need some modification.

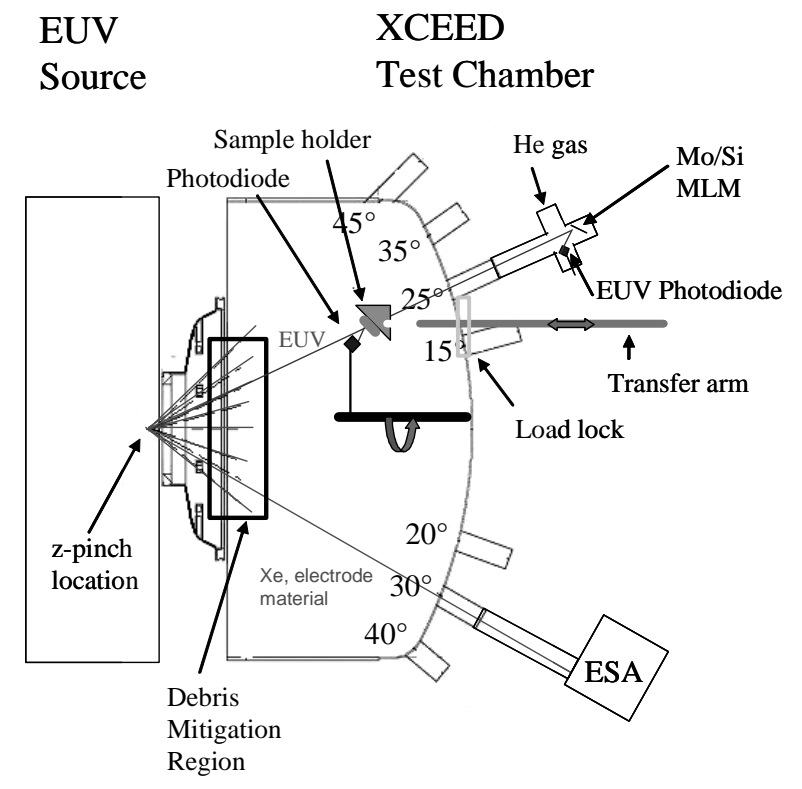

Figure 1. Schematic of the Xtreme Commercial EUV Exposure Device (XCEED)

The Spherical Sector Energy Analyzer (ESA) is a well-characterized diagnostic capable of measuring ion energy and discriminating by charge state. These experiments use a Comstock model AC- $902^{\mathrm{TM}}$ with dual microchannel plate detectors from Burle Corporation (model CP- $618 \mathrm{C}^{\mathrm{TM}}$ ). The analyzer has line-of-sight access to the source through $23 / 4$ in. $\mathrm{CF}$ half nipples positioned at angular intervals of $5^{\circ}$ from $15^{\circ}$ to $45^{\circ}$ from the centerline of the pinch. Access at $0^{\circ}$ is impeded by the beam stop of the debris tool. Current experiments are performed at $20^{\circ}$ and $30^{\circ}$ angles. Data acquisition for the ESA is triggered by the rising light signal from this photodiode. The ESA is mounted on a bellows connection to the chamber for 3 dimensional pointing control. A $1 \mathrm{~mm}$ orifice located proximal to the ESA limits flow to the diagnostic. The entrance orifice to the ESA deflector section is $3 \mathrm{~mm}$ in diameter and total distance traversed by the ions from the source to the microchannel plates is $150 \mathrm{~cm}$.

The samples investigated consist of four identical $1 / 2$ " by $1 / 2$ " pieces of a Si/Mo multilayer mirror (MLM). The multilayer, "Prep 1", is optimized for $5^{\circ}$ operation with 50 bilayer pairs with a period thickness of $6.95 \mathrm{~nm}$ and a gamma of 0.4 , and has a $2.3 \mathrm{~nm}$ ruthenium capping layer. It was prepared by Saša Bajt at Lawrence Livermore National 
Laboratory. Previous ML1 samples were characterized before exposure, after exposure to 500,000 shots in the ETS LPP source at Sandia National Laboratories, and after 10 million shots in XCEED.

The samples were exposed for a maximum of 40 million shots in XCEED over approximately 43 hours running at a repetition rate of $256 \mathrm{~Hz}$. The exposures were done with debris mitigation active for the EUV source system. The plan called for a sample to be removed at 2 million, 10 million, 20 million, and 40 million shots. However, a problem removing one of the sample holders at 20 million shots forced two samples to be exposed for the full 40 million shots. Data from the two photodiodes was taken roughly every 15 minutes throughout the exposures.

Details of the experimental setup and characterization of the measured debris spectrum from the source can be found elsewhere in these proceedings ${ }^{1}$.

\section{RESULTS AND DISCUSSION}

Based on the data from the reference photodiode, the light output from the source varied with time. Oscilloscope traces of the reference photodiode signal near the beginning of the exposures and near the end are shown in Figure 2. The integrated area of the signals vs. shot number is shown in Figure 3. The total signal initially decreases during the first half of the exposures, but increases steadily toward the end. At 20 million shots, it was observed that the electrode appeared to be glowing - indicating it was excessively hot. The source was powered down at that time for 30 seconds to see that it cooled off. The light emitted by the electrode faded quickly, and the exposures were resumed to minimize any discontinuity in the exposures. However, it is possible that after the electrodes cooled something changed that increased the EUV output. For example, perhaps the shape or size of the pinch was altered.

The signal from the photodiode looking at the samples was unfortunately obscured by a very large noise signal with a negative voltage bias. The lack of a usable photodiode signal from the samples precludes the calculation of sample reflectivity vs. exposure time. This is something that is being corrected for the upcoming exposures of palladium samples at grazing incidence.

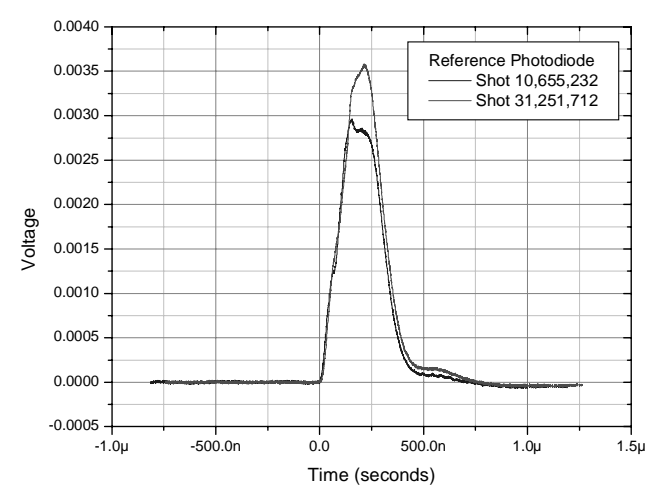

Figure 2. The signal from the reference photodiode during the exposures

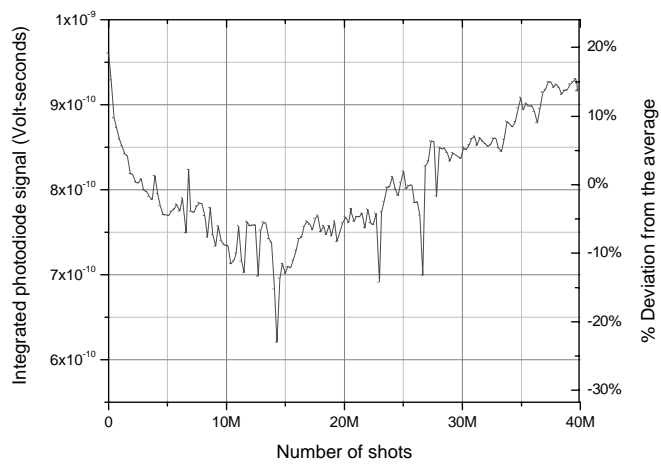

Figure 3. The integrated photodiode signal vs. shot number

Surface roughness can limit reflectivity of surfaces, particularly in the case of a grazing incidence collector. To investigate how the exposures affected the samples in this regard, atomic force microscopy (AFM) was used to measure surface height variations over several different lateral length scales. Scans were done over $5 \times 5,2 \times 2$, $1 \times 1$, and $0.5 \times 0.5$ $\mu \mathrm{m}$ areas. Shown in Figure 4-Figure 8 are $2 \times 2 \mu \mathrm{m}$ AFM scans of the three ML1 samples from the time exposure, together with data on the unexposed sample and one sample previously exposed to 10 million shots. 


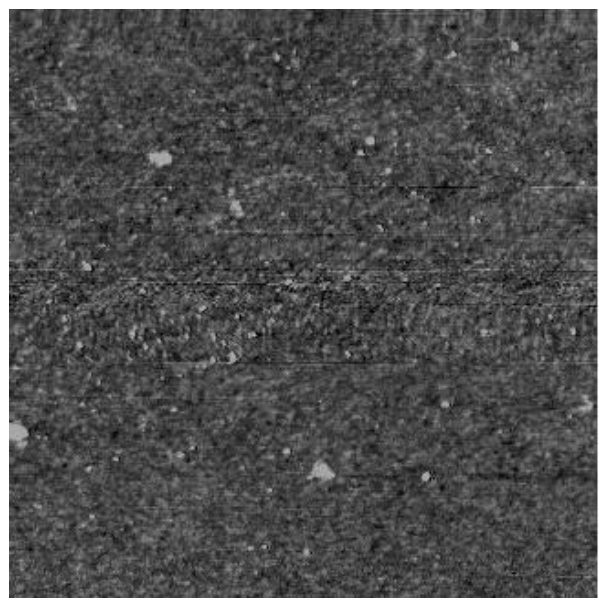

$2.79 \mathrm{~nm}$

Figure 4. AFM $2 \mu \mathrm{m} \times 2 \mu \mathrm{m}$ scan of the unexposed ML1 sample

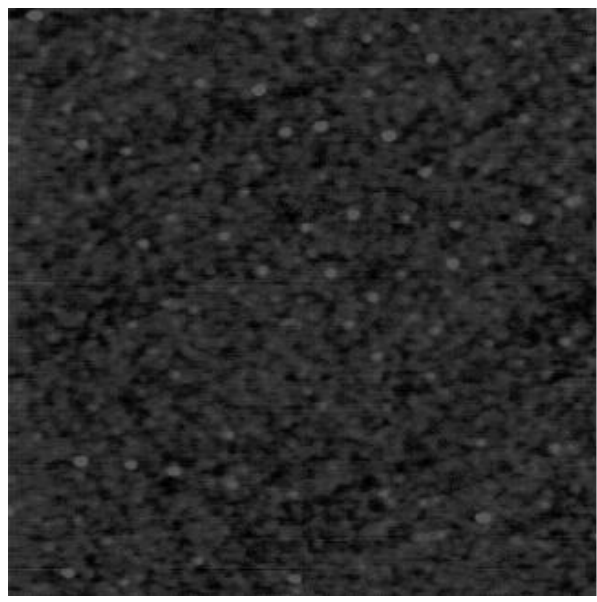

$2.79 \mathrm{~nm}$

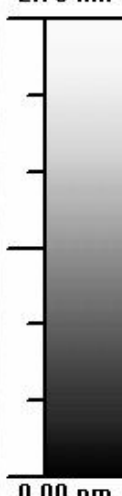

Figure 6. AFM $2 \mu \mathrm{m} \times 2 \mu \mathrm{m}$ scan of the ML1 sample exposed to 10 million shots

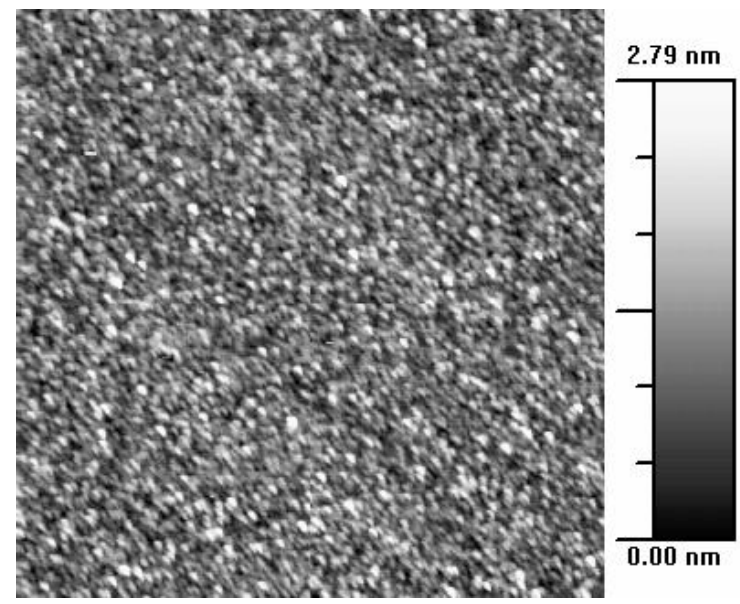

Figure 5. AFM $2 \mu \mathrm{m} \times 2 \mu \mathrm{m}$ scan of the ML1 sample exposed to 2 million shots
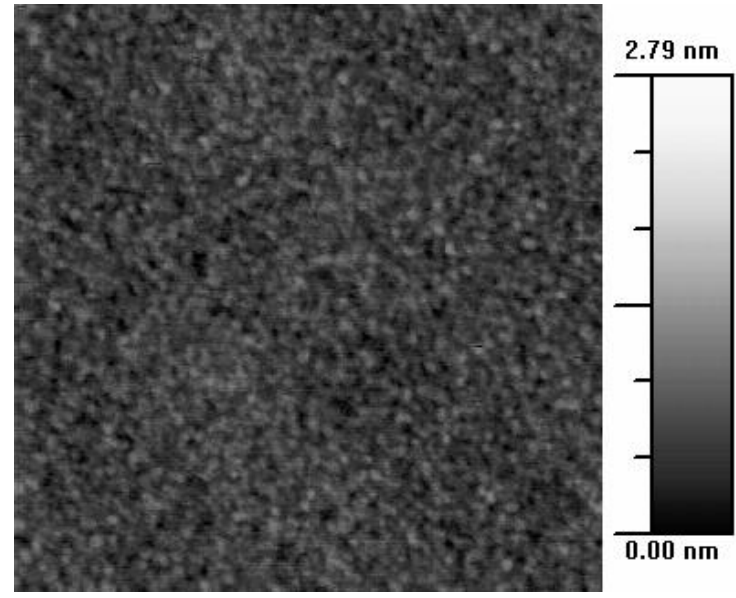

Figure 7. AFM $2 \mu \mathrm{m} \times 2 \mu \mathrm{m}$ scan of the ML1 sample exposed to 11 million shots 


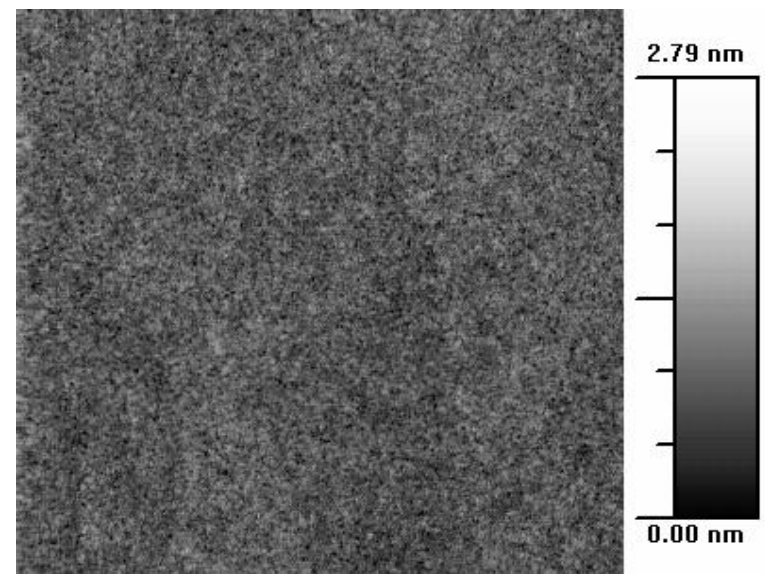

Figure 8. AFM $2 \mu \mathrm{m} \times 2 \mu \mathrm{m}$ scan of the ML1 sample exposed to 40 million shots

In terms of RMS roughness, the most commonly quoted roughness parameter, the roughness increased to $0.53 \mathrm{~nm}$ after 2 million shots, then decreased back to levels near the unexposed sample of 0.20 and $0.23 \mathrm{~nm}$ at 11 million and 40 million shots, respectively. This compares to the unexposed sample with RMS roughness 22 and the previous 10 million shot exposure with RMS roughness of $0.13 \mathrm{~nm}$. It appears that roughness increased quite a bit at first, but as time went on the surface became smoother and the lateral scale of the features became very fine.

Erosion of the samples during exposure was estimated from scanning electron microscopy pictures of the film cross sections. The films are shown in Figure 10-Figure 13. The time-dependent exposure tests showed some erosion of the samples, steadily increasing over the 40 million shots. The 2 million shot sample showed no change in thickness from the unexposed sample. After 11 million shots, a couple of nanometers was eroded. After the full 40 million shots, the thickness had decreased by $6 \mathrm{~nm}$.

Previous experiments showed a larger amount of erosion for the multilayer after exposure to only 10 million shots. The reasons for this difference are not immediately clear. Because the electrode was so completely eroded during the 40 million shots, it is possible that a significant amount of electrode material was deposited throughout the vacuum chamber and on the samples. This could throw off thickness measurements, which can only show net erosion (erosion deposition). This will be investigated further by using x-ray photoelectron spectroscopy to quantify the amount of electrode material present on the sample surfaces. 


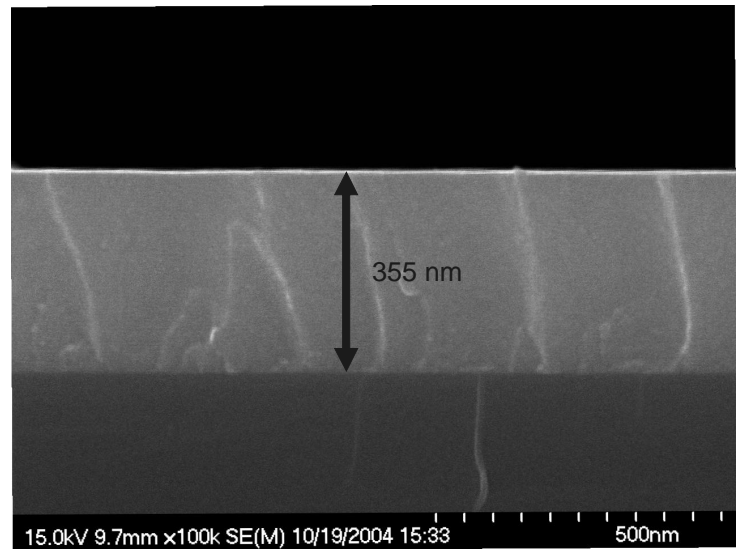

Figure 9. SEM cross section of the unexposed sample

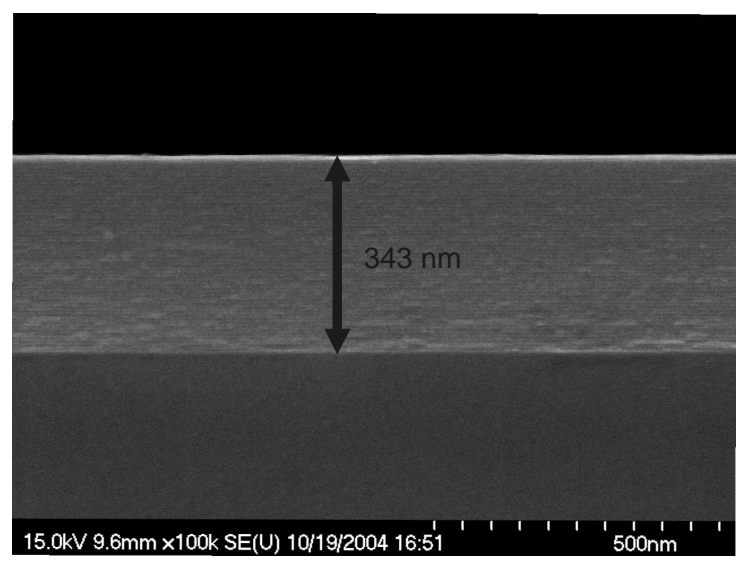

Figure 11. SEM cross section of the sample previously exposed to 10 million shots

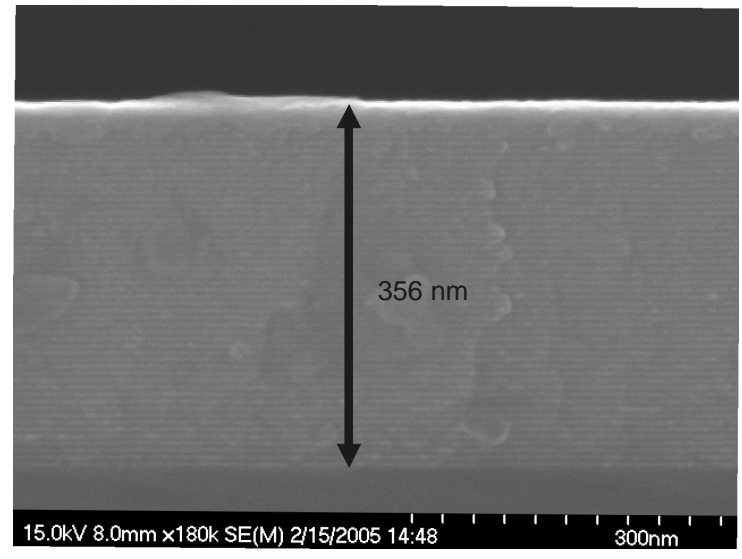

Figure 10. SEM cross section of the sample exposed to 2 million shots

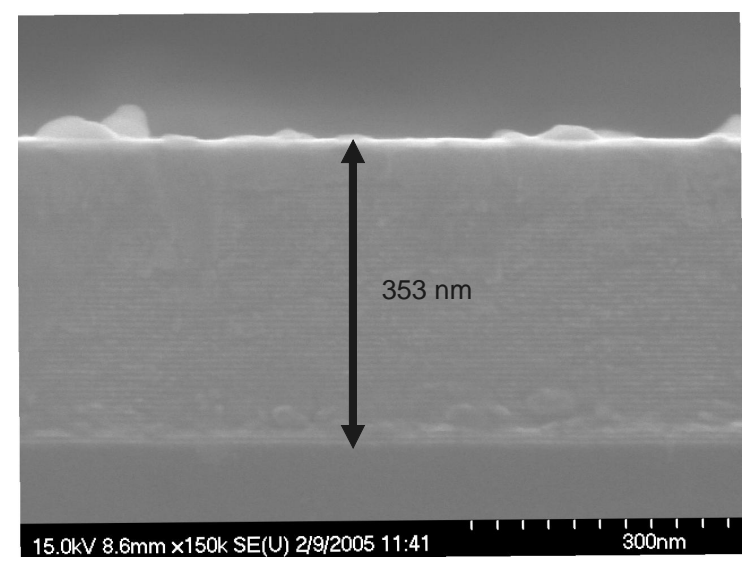

Figure 12. SEM cross section of the sample exposed to 11 million shots 


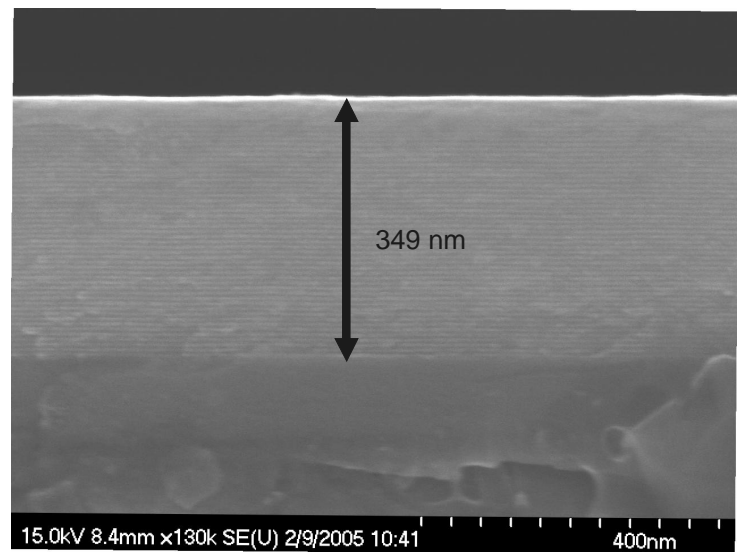

Figure 13. SEM cross section of the sample exposed to 11 million shots

\section{CONCLUSIONS}

Time-dependent exposures were performed in XCEED on four multilayer mirror samples for up to 40 million shots over 43 hours at a repetition rate of $256 \mathrm{~Hz}$. By the end of the exposures, it was discovered that the electrodes had reached the end of their lifetime. During exposure, this could have contributed to variations in the EUV light output over time. The electrode was significantly eroded after the 40 million pulses, and the electrode material was possibly redeposited all over the test chamber. This could affect erosion estimates based on measuring the film thickness in cross-sectional SEM. The time-dependent exposures showed slower erosion than previous 10 million shot exposures of the same samples.

In addition to being eroded, the surface topography of the samples changed with time as well. At first the roughness increased dramatically. As time went on, the RMS roughness returned to approximately the value of an unexposed sample. The sample exposed to 40 million shots shows finer features, suggesting that after many shots the surface is uniformly damaged.

The next time-dependent exposures of four palladium samples at grazing incidence will be performed shortly. These experiments will included a somewhat redesigned photodiode setup to look at the samples, hopefully providing better information on the loss of reflectivity over time.

\section{ACKNOWLEDGEMENTS}

This research was partially carried out in the Center for Microanalysis of Materials, University of Illinois, which is partially supported by the U.S. Department of Energy under grant DEFG02-91-ER45439. This work was partially funded by Intel Components Research and SEMATECH.

\section{REFERENCES}

1. E. L. Antonsen, K. C. Thompson, M. R. Hendricks, et al., "XCEED: XTREME Commercial EUV Exposure Diagnostic Experiment", SPIE Microlithography 2005, San Jose, CA, 2005. 